\section{IS THERE A CORRELATION BETWEEN PRE-OPERATIVE ANXIETY AND PAIN EXPERIENCED DURING PEDIATRIC DENTAL APPLICATIONS?}

O. Baygin ${ }^{1}$, T. Tuzuner ${ }^{1}$, B. Isik², M. Tanrıver ${ }^{1}$, I. Arslan ${ }^{1}$

${ }^{1}$ Department of Pediatric Dentistry, Karadeniz Technical University, Trabzon, ${ }^{2}$ Department of Oral and Maxilla Facial Surgery, Gazi University, Ankara, Turkey

Background and aim: Pain in children may occur resultant of various diseases, trauma and medical attempts. Nowadays dental applications as one of medical attempts are considered to be painful. This pain induces anxiety and affects behaviours during treatment. Especially, majority of children are extremely anxious and feel as if they will experience pain. As a result cooperation problems are the main factor for the failure of procedures in treatment sessions. There are many methods for evaluating pain-anxiety and they can be classified as behavioral, physiologic, projective and psychometric techniques. It is aimed in this study to evaluate the correlation between the level of anxiety before treatment and pain experienced during the procedures.

Method: Frankl Scale, The Children's Dental Fear Picture Test, Spielberg State-Trait Anxiety Inventory before anaesthesia and dental applications and The Wong-Baker Pain Scale, Visual Analog Scale after the procedures were implemented to randomly selected 100 child patients between $6-12$ years old referred to the Pediatric Dental Clinic of Karadeniz Technical University Faculty of Dentistry.

Result: Correlation was found between scales applied before and after the procedures.The patients who exhibited higher anxiety scores to pre-procedural scales also resulted in significantly higher pain scores to post-procedural scales ( $p<$ 0.05).

Conclusion: It can be concluded that, children who feel highly anxious before dental treatment and have experienced more pain to this anxiety level could be related with their previous dental experience and personality characteristics. Keeping the patient with minimal anxiety will facilitate the dental procedures for both the patient and the clinical.

\section{INTRANASAL LIDOCAINE AND MIDAZOLAM FOR PROCEDURAL SEDATION IN CHILDREN}

A. Chiaretti, F. Pierri, G. Barone, D. Rigante, A. Ruggiero, V. Giorgio, V. D’Andrea, R. Riccardo

Pediatric Sciences, Catholic University of Sacred Heart, Gemelli Hospital, Rome, Italy

Objective: To evaluate the feasibility of a sedation protocol based on Lidocaine spray and Intranasal Midazolam (INM) administered by Mucosal Atomizer Device (MAD) in uncooperative children undergoing minor painful procedures or diagnostic investigations.

Patients and design: Fourty-six children, aged 6-60 months, underwent INM $(0.5 \mathrm{mg} / \mathrm{kg})$ by MAD after delivering a puff of Lidocaine spray (10 mg per puff) before the execution of various procedures, such as peripheral line placements, venipunctures, intramuscular injections, heart ultrasound examinations, computed tomographic scans, auditory tests or dental manipulation. A questionnaire was designed to evaluate the effect of sedation in performing the procedures. Statistical analysis was performed to compare sedation times with age and weight of children tested.

Results: The degree of sedation achieved by INM allowed all procedures to be completed without any additional drugs. Premedication with Lidocaine spray prevented any nasal discomfort or pain related to INM. The mean duration of sedation effect was 23.1 minutes. No significant side effects could be recorded in the cohort of children studied. The degree of appreciation for both medical staff and children's parents was good. A significant correlation of effect start and end time was found only with age.

Conclusions: This study showed that the association of Lidocaine spray and atomized INM is safe and effective to obtain children's short-term sedation and to easily perform all the procedures. 